\title{
Fluorescence spectroscopic studies on binding of a flavonoid antioxidant quercetin to serum albumins
}

\author{
BEENA MISHRA, ATANU BARIK, K INDIRA PRIYADARSINI* and HARI MOHAN \\ Radiation Chemistry \& Chemical Dynamics Division, Bhabha Atomic Research Centre, Trombay, \\ Mumbai 400 085, India \\ e-mail: kindira@apsara.barc.ernet.in
}

MS received 3 December 2004; revised 24 March 2005

\begin{abstract}
Binding of quercetin to human serum albumin (HSA) was studied and the binding constant measured by following the red-shifted absorption spectrum of quercetin in the presence of HSA and the quenching of the intrinsic protein fluorescence in the presence of different concentrations of quercetin. Fluorescence lifetime measurements of HSA showed decrease in the average lifetimes indicating binding at a location, near the tryptophan moiety, and the possibility of fluorescence energy transfer between excited tryptophan and quercetin. Critical transfer distance $\left(R_{o}\right)$ was determined, from which the mean distance between tryptophan-214 in HSA and quercetin was calculated. The above studies were also carried out with bovine serum albumin (BSA).
\end{abstract}

Keywords. Human serum albumin; bovine serum albumin; quercetin; energy transfer; binding constant.

\section{Introduction}

Serum albumins are abundantly found in blood plasma and are often termed transport proteins. ${ }^{1-4}$ They are circulated in the body several times and act as carriers for numerous exogenous and endogenous compounds. The most popularly studied albumins are bovine serum albumin (BSA) and human serum albumin (HSA). Both BSA and HSA have very high conformational adaptability to a great variety of ligands. ${ }^{4}$ Two main approaches have been adopted in the ligand-protein binding studies. While some groups have studied the in vivo consequences of binding of drugs and other metabolites to serum albumins, ${ }^{5-7}$ others have examined the binding mechanism using absorption, fluorescence, circular dichroism etc. ${ }^{8-19}$ Based on such studies, information on the binding process of many exogenous ligands like long chain fatty acids, amino acids, metals, drugs, bilirubin etc have been reported at the molecular level. It has also been considered that such binding can increase the solubility of ligands and there are reports that the toxicity of some ligands like bilirubin decreased on bonding to albumins. ${ }^{4,8,9}$

In general, albumins are characterized by low tryptophan and high cystine contents. ${ }^{1-4}$ BSA and

*For correspondence
HSA mainly differ in tryptophan content. Except for this, the amino acid compositions of the two proteins are nearly the same. HSA has one tryptophan group at the 214 position, while BSA has tryptophan groups at 134 and 212 position. Primarily, three domains and six principal binding sites have been identified for several important biomolecules. ${ }^{4,17,20,21}$

Antioxidants are a class of biomolecules primarily used to reduce oxidative stress. Quercetin $\left(3,3^{\prime}, 4^{\prime}, 5,7-\right.$ pentahydroxyflavone) is a flavonoid antioxidant which has been found in a wide variety of plant products. It is an excellent free radical scavenger and a metal chelator. It is one of the most potent bioflavonoids studied and possesses a variety of pharmacological activities including anti-inflammatory, antineoplastic, cardioprotective and anticancer activities. ${ }^{22,23}$ Quercetin is weakly soluble in water and its solubility increases in lipids and proteins. Recently, there have been a few reports on the interaction of quercetin with serum albumins. ${ }^{24-32}$ Boulton et al determined the association constant of quercetin binding to HSA by radiolabelling studies and suggested the binding at IIA domain. ${ }^{24}$ Dufour and Dangles suggested that flavonoids display moderate affinities for albumins while flavones and flavonols exhibit higher efficiency. ${ }^{32}$ By fluorescence energy transfer studies, Sytnik and Litvinik reported that flavonols bind in the IIIA and IIA sub domain of HSA. ${ }^{33}$ Us- 
ing steady state emission and absorption measurements, Sengupta et al studied the binding of quercetin to HSA and proposed the possibility of fluorescence energy transfer. ${ }^{28-30}$ The energy transfer efficiency between several flavonoids and HSA were studied by Bi et al. ${ }^{31}$ In this present paper using fluorescence quenching and energy transfer studies, we report the association constants for the binding of quercetin to BSA and HSA. The probable mean distance between tryptophan and quercetin for these proteins is estimated, with the object of identifying the most probable site of binding for quercetin.

\section{Experimental}

\subsection{Chemicals}

BSA and HSA (purity 99\%, crystallized and lyophilized), tryptophan and quercetin from Sigma were used as received. Solutions were prepared in nanopure water from a Millipore Milli Q system. Wherever necessary spectrograde solvents from Spectro Chem, India were used.

\subsection{Absorption and fluorescence studies}

Absorption spectra were recorded on a Jasco V530 spectrophotometer and fluorescence spectra on a Hitachi F-4010 fluorimeter.

Fluorescence quantum yields of HSA and BSA were determined by using tryptophan as the reference with known $\Phi_{\mathrm{R}}$ of $0 \cdot 13$ in water. ${ }^{34}$ Reference and albumin samples were excited at $290 \mathrm{~nm}$, maintaining nearly the same absorbance, and emission spectra were recorded from 300 to $450 \mathrm{~nm}$. The area under the emission spectrum was integrated using the software available in the instrument, and the quantum yield calculated according to the following,

$$
\frac{\Phi_{\mathrm{S}}}{\Phi_{\mathrm{R}}}=\frac{A_{\mathrm{S}}}{A_{\mathrm{R}}} \times \frac{(\mathrm{Abs})_{\mathrm{R}}}{(\mathrm{Abs})_{\mathrm{S}}} \times \frac{n_{\mathrm{S}}^{2}}{n_{\mathrm{R}}^{2}} .
$$

Here $\ddot{O}_{\mathrm{S}}$ and $\ddot{O}_{\mathrm{R}}$ are the fluorescence quantum yields of sample and reference respectively. $A_{\mathrm{S}}$ and $A_{\mathrm{R}}$ are the area under the fluorescence spectra of sample and reference respectively, $(\mathrm{Abs})_{\mathrm{S}}$ and $(\mathrm{Abs})_{\mathrm{R}}$ are their respective optical densities at the wavelength of excitation, and $n_{\mathrm{S}}$ and $n_{\mathrm{R}}$ are the values of refractive index for the respective solvents used for them.

\subsection{Time-resolved fluorescence studies}

Fluorescence lifetimes were measured using a timecorrelated single-photon counting spectrometer model 199 from Edinburgh Instrument, UK, details of which are available in O'Connor and Phillips. ${ }^{35}$ Decay curves were fitted using a nonlinear iterative least square fit method using the following,

$$
G(t)=\sum_{i} B_{i} \exp \left(-t / \tau_{i}\right)
$$

$G(t)$ is the fitted decay curve usually assumed to be a sum of exponentials, where $B_{i}$ is the pre-exponential factor for the $i$ th component and $\hat{o}_{i}$ is the corresponding fluorescence lifetime. The bi-exponential or tri-exponential nature of the fluorescence decays was judged from the reduced $\chi^{2}$ values $(1 \cdot 00-1 \cdot 15)$ and the distribution of the weighted residuals (random) among the data channels. ${ }^{33}$

\subsection{Preparation of protein-quercetin complexes}

As quercetin is insoluble in water, a stock solution of $10 \mathrm{mM}$ quercetin in methanol was first prepared and then diluted with $5 \mathrm{mM}$ aqueous $\mathrm{Na}_{2} \mathrm{HPO}_{4}$ solution to get a clear solution of $100 \mu \mathrm{M}$ quercetin, such that the methanol content in the solution did not exceed $1 \%$. Albumin was dissolved in $32.4 \mathrm{mM}$ $\mathrm{Na}_{2} \mathrm{HPO}_{4}$ and $7.5 \mathrm{mM} \mathrm{KH} \mathrm{PO}_{4}$. These two solutions of albumin and quercetin were mixed to obtain the required concentration of albumin and quercetin. The $\mathrm{pH}$ of the solution after mixing was 7.4. Enough care was taken to see that all the solutions contained the same concentrations of buffers and identical $\mathrm{pH}$ was maintained all through. This system was used to estimate the binding constant of quercetin with albumin. For fluorescence studies, the absorbance at the excitation wavelength was kept at $0 \cdot 2$ to $1 \cdot 0$. Alternatively, quercetin-serum albumin complex was also prepared by dialyzing a $1: 4$ mixture of serum albumin and quercetin mixture in phosphate buffer $(10 \mathrm{mM})$ for $20 \mathrm{~h}$ against $20 \mathrm{mM}$ phosphate buffer $(\mathrm{pH}$ 7) with buffer change every $4 \mathrm{~h}$. These solutions were used for the estimation of energy transfer parameters and other photo-physical properties of quercetin.

\section{Results and discussion}

The absorption spectrum of quercetin in presence of either BSA or HSA showed significant red shift. 
Figure 1 shows the spectral changes on interactions of varying concentrations of quercetin with $30 \mu \mathrm{M}$ HSA. The corresponding spectra for BSA are not shown as they look similar and only the data are presented. The spectral changes are attributed to the formation of a complex between albumin and quercetin, as given below,

$$
n \text { quercetin }+\mathrm{SA} \rightleftharpoons \text { complex. }
$$

The association constant $K$ for the above equilibrium is represented by,

$$
K=\frac{[\text { complex }]}{[\text { SA }][\text { quercetin }]^{n}} \text {. }
$$

Here SA is either HSA or BSA, $K$ is the binding constant and $n$ the number of binding sites.

At a wavelength where the albumins and unbound quercetin have minimum absorption and there is a maximum change in the absorbance on binding, the absorbance changes $(\Delta A)$ were followed at different concentrations of quercetin and plotted according to the Scatchard equation (5). Accordingly $\Delta A$ at 425 and $405 \mathrm{~nm}$ for HSA and BSA respectively were followed.

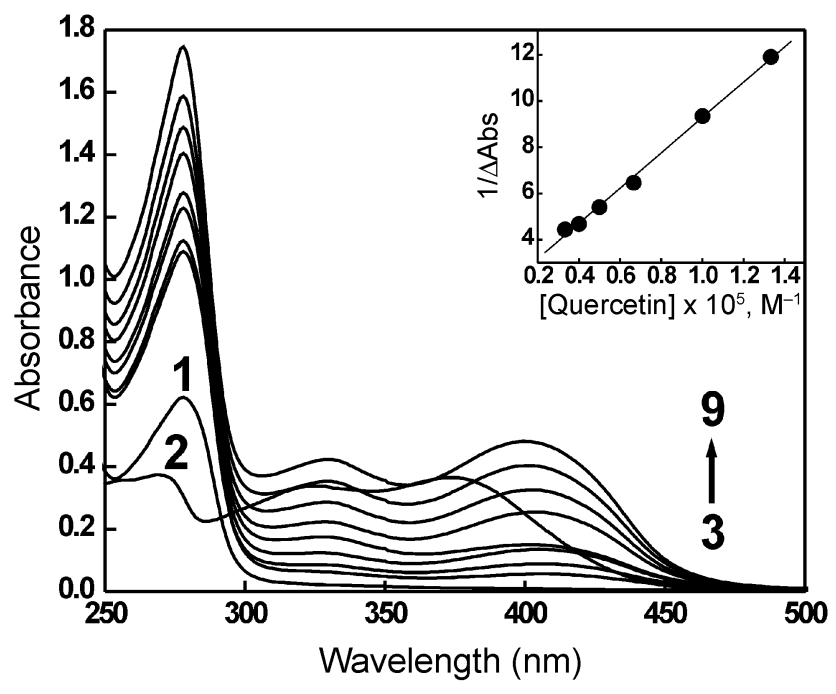

Figure 1. Absorption spectra of aqueous solutions containing $30 \mu \mathrm{M}$ HSA in presence of different concentrations of quercetin from $2 \cdot 5 \mu \mathrm{M}$ to $30 \mu \mathrm{M}$ at $\mathrm{pH} 7.4$. Spectra 1 and 2 represent $30 \mu \mathrm{M}$ HSA blank and $25 \mu \mathrm{M}$ quercetin blank respectively. Spectra 3-9 correspond to $2 \cdot 5,5 \cdot 0,7 \cdot 5,10,20,25,30 \mu \mathrm{M}$ quercetin in presence of $30 \mu \mathrm{M}$ HSA. Inset shows double reciprocal linear plot according to the Scatchard equation, (5).

$$
\frac{1}{\Delta A}=\frac{1}{n \Delta \varepsilon[\mathrm{SA}]}+\frac{1}{n \Delta \varepsilon K[\mathrm{SA}][\text { quercetin }]}
$$

Here $\Delta \varepsilon$ is the molar extinction coefficient at 425 and $405 \mathrm{~nm}$ respectively for quercetin bound to HSA and BSA and $[\mathrm{SA}]$ is the concentration of either HSA or BSA. According to (5), the double reciprocal plot for the above changes is given in the inset of figure 1. From this, the values of $K$ and $n$ for binding of quercetin to HSA were $3 \cdot 1 \pm 0 \cdot 1 \times 10^{4} \mathrm{M}^{-1}$ and $1 \cdot 1 \pm 0 \cdot 1$ respectively. The corresponding values of $K$ and $n$ for BSA were determined as $3.5 \pm 0.6 \times$ $10^{4} \mathrm{M}^{-1}$ and $1 \cdot 0 \pm 0 \cdot 1$ respectively. The extinction coefficients of $1.01 \times 10^{4} \mathrm{M}^{-1} \mathrm{~cm}^{-1}$ at $405 \mathrm{~nm}$ and $1.6 \times 10^{4} \mathrm{M}^{-1} \mathrm{~cm}^{-1}$ at $425 \mathrm{~nm}$ respectively were estimated for quercetin bound to BSA and HSA, which were used for all further calculations.

The fluorescence quantum yields of HSA and BSA were estimated to be 0.054 and 0.101 respectively according to the procedure discussed above. Due to the presence of two tryptophan groups, BSA shows almost double the fluorescence quantum yield. In the presence of quercetin, the protein fluorescence was found to decrease, and this quenching in fluorescence was used to estimate the binding constant $K$. For these studies, solutions containing $30 \mu \mathrm{M}$ serum albumin and 2.5 to $20 \mu \mathrm{M}$ quercetin at $\mathrm{pH} 7.4$ were excited at $290 \mathrm{~nm}$ and the emission was monitored in the 300 to $450 \mathrm{~nm}$ region. At quercetin concentrations above $20 \mu \mathrm{M}$, due to its own absorption (OD 0.2) at $290 \mathrm{~nm}$, part of the light may be absorbed directly by quercetin, thus reducing the formation of the excited states of albumin and causing decrease in albumin fluorescence. Hence data above $20 \mu \mathrm{M}$ is not used for binding constant measurements. Figure 2 shows the fluorescence spectra of HSA in presence of different concentrations of quercetin. It can be seen that the fluorescence intensity at $342 \mathrm{~nm}$ decreases in presence of quercetin. This change in fluorescence intensity at $342 \mathrm{~nm}$ is used to estimate $K$ and $n$ for the binding of quercetin to serum albumin according to the procedure given earlier $^{11,18}$ and also from the following equation,

$$
\log \left(\frac{F_{o}-F}{F}\right)=\log K+n \log [\text { quercetin] }
$$

Here $F_{o}$ and $F$ are the fluorescence intensity from the fluorophore, albumin, at $342 \mathrm{~nm}$ in the absence and the presence of different concentrations of 
quercetin respectively. The inset of figure 2 shows a linear plot for $\left(\log \left(F_{o}-F\right) / F\right)$ vs $(\log$ [quercetin]) for HSA. From this, according to (6), the values of $K$ and $n$ were estimated to be $2.3 \pm 0.6 \times 10^{4} \mathrm{M}^{-1}$ and $1 \cdot 1 \pm 0 \cdot 2$ respectively for HSA and the corresponding values for BSA were $4.85 \pm 0.15 \times 10^{5} \mathrm{M}^{-1}$ and $1.19 \pm 0.05$ respectively (figure not shown). From the above studies, it is concluded that there is a single binding site for quercetin in HSA and BSA. The above determined binding constants by absorption and fluorescence methods agree well for HSA but differ considerably with those for BSA. This is because of the presence of two tryptophan moieties in BSA, binding of the probe to the protein influences the fluorescence from one of the tryptophan moieties, while the other is unaffected, causing errors in the estimation of binding constant by the fluorescence method for BSA. In case of HSA, due to the presence of a single tryptophan moiety, such errors are not expected and the fluorescence quenching corresponds to binding near the tryptophan moiety only. The binding constants reported in the literature for quercetin and HSA vary from $10^{5}$ to $10^{4} \mathrm{M}^{-1}$ $\left(2.67 \times 10^{5}, 1.46 \times 10^{4}\right.$ and $1.1 \times 10^{5} \mathrm{M}^{-1}$ respectively in references 24,27 and 28 ). Our estimated values of binding constant are within the limits of the reported data. The above observed protein fluorescence quenching by quercetin was examined fur-

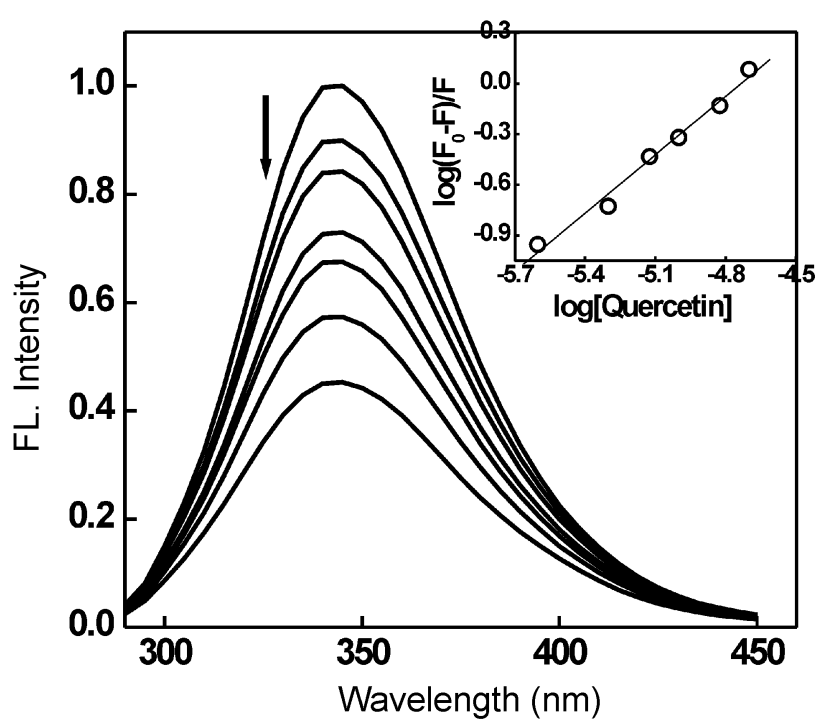

Figure 2. Fluorescence spectra of aqueous solutions of $30 \mu \mathrm{M}$ HSA in presence of different concentrations of quercetin $0,2 \cdot 5,5,7 \cdot 5,10,15$ and $20 \mu \mathrm{M}$ at $\mathrm{pH} 7 \cdot 4$, excitation wavelength, $290 \mathrm{~nm}$. Inset shows the linear plot for $\log \left(F_{o}-F\right) / F$ vs $\log$ [quercetin] according to (6). ther for the possibility of excitation energy transfer between the tryptophan and the bound quercetin as observed in the case of several other fluorophores.

Since the absorption spectrum of bound quercetin efficiently overlaps with the fluorescence emission spectrum of the tryptophan in HSA, the decrease in tryptophan emission may be due to ground-state quenching (static quenching) of tryptophan fluorescence by quercetin or by inner filtration effect, or it may be due to direct energy transfer from singlet excited state of tryptophan to quercetin (dynamic quenching). In order to verify this, time-resolved florescence studies were carried out.

Time-resolved fluorescence lifetime measurements were carried out for BSA and HSA in the absence and presence of quercetin. For this, albumin samples of $30 \mu \mathrm{M}$ were excited at $290 \mathrm{~nm}$ and the emission intensity collected at $340 \mathrm{~nm}$. Fluorescence decay for HSA and BSA could be fitted to multiple exponential functions. HSA shows biexponetial fit (figure 3a), with relative fluorescence lifetimes of $2.34 \mathrm{~ns}(39 \%)$ and $6.33 \mathrm{~ns}(61 \%)$ and average life-

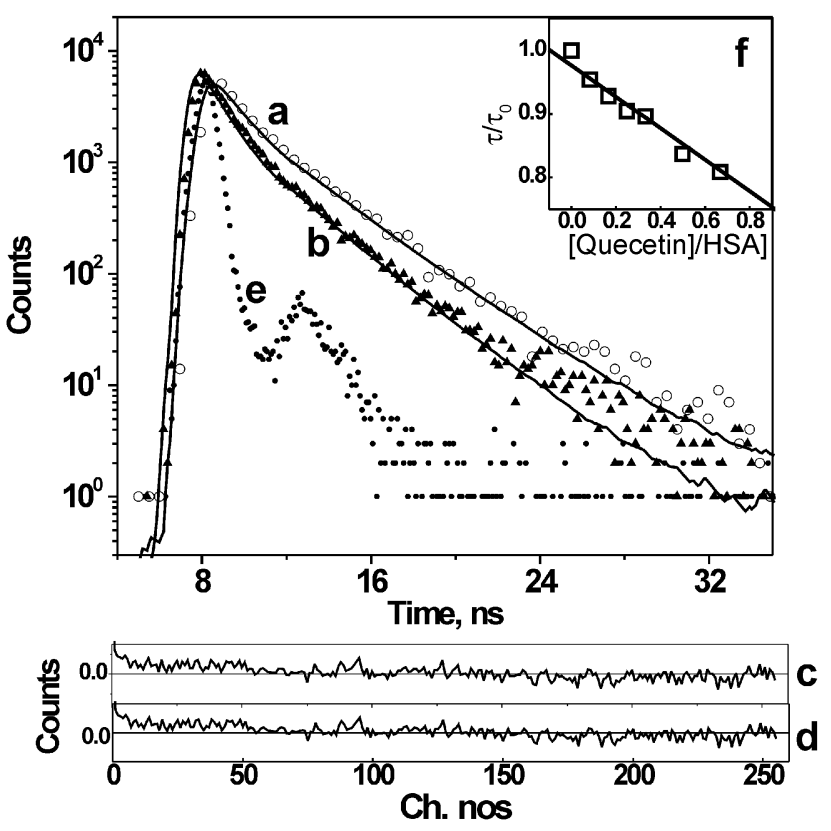

Figure 3. Fluorescence decay curve along with the fitted curve (smooth line) showing the decay of (a) $30 \mu \mathrm{M}$ HSA and (b) $30 \mu \mathrm{M} H S A+30 \mu \mathrm{M}$ quercetin, fitted to a biexponential function after excitation at $290 \mathrm{~nm}$ and emission at $340 \mathrm{~nm}$. c and d represent the distribution of the residuals for curves $a$ and $b$ respectively. The instrument response is shown as curve (e). Inset (f) shows linear variation of the average fluorescence lifetime ratio as a function of moles of quercetin bound per mole of protein. 
time of $4.76 \mathrm{~ns}\left(\tau_{\mathrm{o}}\right)$, while the data for BSA show triple exponential decay, with relative fluorescence lifetimes of $3.84 \mathrm{~ns}(32.5 \%), 0.13 \mathrm{~ns}(2.5 \%)$ and $7.21 \mathrm{~ns}(65 \%)$ and average lifetime of $5.94 \mathrm{~ns}$ (figure not shown). In the literature, there are several reports with widely different values on the fluorescence lifetimes of both HSA and BSA. Our values fall within the recent estimations of lifetimes for HSA and BSA. When $30 \mu \mathrm{M}$ quercetin was added to these samples, the fluorescence lifetime decreased. For HSA, the fluorescence lifetimes were $5.87 \mathrm{~ns}$ $(44.6 \%)$ and $2.02 \mathrm{~ns}(55.4 \%)$ with average lifetime of $3.745 \mathrm{~ns}$ (figure $3 \mathrm{~b}$ ). It can be seen that the decay remains biexponential and both the components decrease. Also their relative percentage does not show any systematic variation. Therefore only the average lifetime $(\tau)$ was compared in presence of different concentrations of quercetin. The linear plot for the variation of $\tau / \tau_{\mathrm{o}}$ as a function of moles of quercetin per mole of HSA is given as inset (e) in figure 3. These studies suggest that quercetin binds to HSA at a location near the tryptophan moiety and there is a possibility of fluorescence energy transfer between the excited tryptophan and quercetin. To further support our results, the above solutions were excited at $290 \mathrm{~nm}$ and the fluorescence from 450 to $600 \mathrm{~nm}$ region due to quercetin fluorescence was monitored using a suitable cut-off filter to avoid interference from other sources. Here, if energy transfer

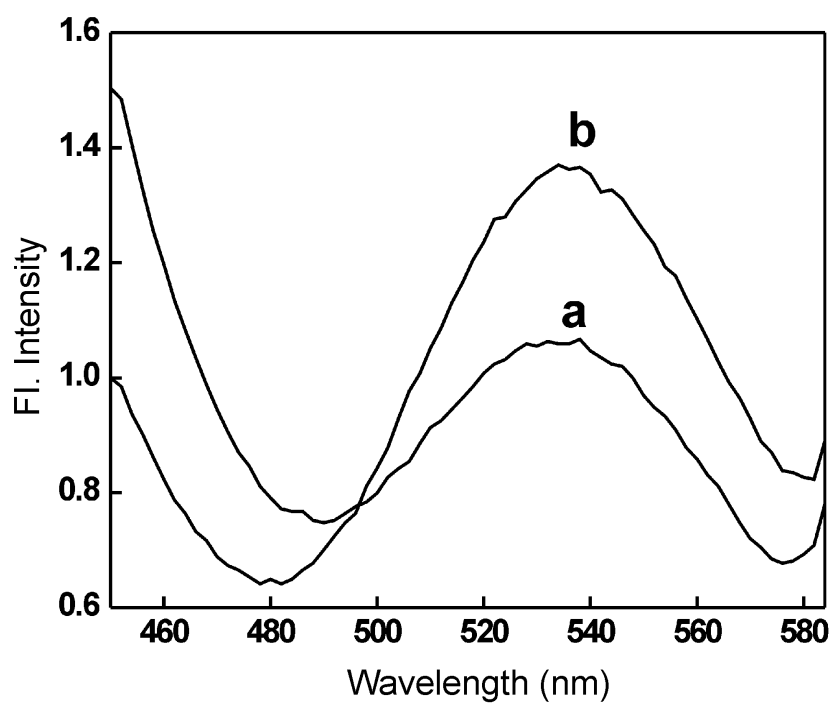

Figure 4. Fluorescence spectra showing increase in fluorescence intensity from quercetin $[(\mathrm{a})=15 \mu \mathrm{M},(\mathrm{b})=$ $30 \mu \mathrm{M}]$ in presence of $30 \mu \mathrm{M}$ HSA obtained after energy transfer from the excited tryptophan in HSA. Excitation wavelength $=290 \mathrm{~nm}$. exists between excited tryptophan and quercetin, the primarily excited tryptophan molecules can induce fluorescence from quercetin. Figure 4 gives fluorescence spectra from 15 and $30 \mu \mathrm{M}$ quercetin in presence of $30 \mu \mathrm{M}$ HSA, after excitation at $290 \mathrm{~nm}$ and energy transfer from the excited tryptophan. Under the same conditions, blank quercetin in the absence of HSA does not show any detectable fluorescence, confirming that the observed fluorescence in these systems is due to binding and subsequent energy transfer. Taking together all the above facts, it is concluded that the quenching of the tryptophan is due to non-radiative energy transfer between Trp-214 and quercetin rather than direct perturbation of the protein environment by quercetin. The energy transfer parameters and the mean distance between the tryptophan moiety in HSA and the quercetin binding site was determined by applying Förster's theory of resonance energy transfer. ${ }^{31,32}$

For energy transfer studies, a quercetin-protein complex was prepared by dialysis as described in the experimental section. The amount of quercetin bound to the complex was determined by using the extinction coefficient at 425 or $405 \mathrm{~nm}$. From this, it was observed that $28 \mu \mathrm{M}$ quercetin binds to $30 \mu \mathrm{M}$ HSA and $30 \mu \mathrm{M}$ quercetin binds to $30 \mu \mathrm{M}$ BSA. These samples were used to estimate energy transfer parameters between albumin and quercetin.

The efficiency of energy transfer $(E)$ is related to the distance $r(\AA)$ between the donor and acceptor by

$$
E=\frac{R_{0}^{6}}{R_{0}^{6}+r^{6}}=1-\frac{\tau}{\tau_{0}}=1-\frac{\phi}{\phi_{0}} .
$$

The energy transfer rate $\left(k_{e t}\right)$ is defined as

$$
k_{e t}=\frac{1}{\tau_{o}}\left(\frac{R_{o}}{r}\right)^{6} \text {. }
$$

Here $\tau_{o}$ is the fluorescence lifetime of the unquenched protein and $R_{o}$ is defined as critical transfer distance at which the transfer efficiency equals $50 \%$ or the fluorescence of donor is quenched by $50 \%$. $R_{o}$ is calculated by using the relation, ${ }^{32}$

$$
R_{o}=9.79 \times 10^{3}\left(\kappa^{2} \Phi_{o} J n^{-4}\right)^{\frac{1}{6}} \AA
$$

where $J$ is the spectral overlap integral between the donor emission and the acceptor absorption, and $\kappa^{2}$ 
is the orientation factor between the emission of the dipole of the donor and the absorption dipole of the acceptor, which is generally $2 / 3$ for isotropic donor and acceptor, $\Phi_{\mathrm{o}}$ is the quantum yield of the donor (the $\Phi_{0}$ of tryptophan in HSA and BSA) and $n$ is the refractive index of the medium. The spectral overlap integral $(J)$ between the donor emission spectrum and the acceptor absorption spectrum was determined by,

$$
J(\lambda)=\left(\int_{0}^{\infty} F_{D} \varepsilon_{A} \lambda^{4} \mathrm{~d} \lambda\right) /\left(\int_{0}^{\infty} F_{D} \mathrm{~d} \lambda\right) .
$$

Here $F(\lambda)$ and $\varepsilon(\lambda)$ represent the fluorescence intensity of the donor and the molar extinction coefficient of the acceptor respectively, at the wavelength $\lambda$.

Figure 5 gives the fluorescence spectra of HSA and the absorption spectra of protein-bound quercetin, the overlap area was integrated with the software available with origin $6 \cdot 1$ and the spectral overlap integral $J$ was estimated to be $1.6 \times 10^{-14} \mathrm{M}^{-1} \mathrm{~cm}^{3}$. Using the above parameters and according to (9), the value of $R_{o}$ was estimated to be $23.51 \AA$. Similarly for the BSA-quercetin complex, $J$ value was estimated to be $1.05 \times 10^{-14} \mathrm{M}^{-1} \mathrm{~cm}^{3}$ and the value of $R_{o}$ was calculated to be $24 \cdot 26 \AA$.

Under these conditions, donor fluorescence was measured both in absence and presence of quercetin. From the integrated area, the fluorescence quantum yields $\phi_{\mathrm{o}}$ and $\phi$ were estimated for the albumin and albumin-quercetin complex respectively. Using these data and the above determined $R_{o}$ value, and

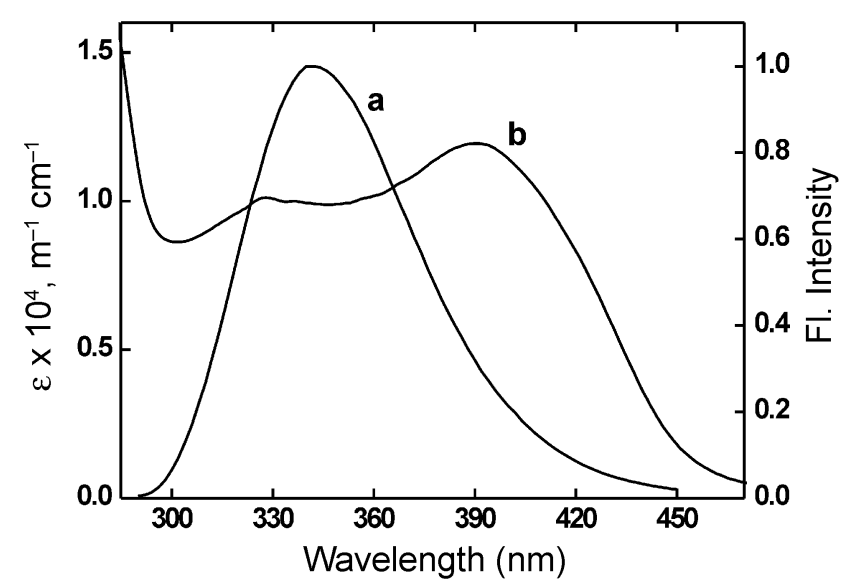

Figure 5. Spectra showing overlap between the fluorescence spectrum of HSA (spectrum a) and the absorption spectrum of HSA-quercetin complex (spectrum b). The overlap integral $J$ is calculated according to (10). according to (7), energy transfer efficiency was estimated to be 0.55 and 0.56 for BSA and HSA respectively. From this, $k_{e t}$ and the distance between the quercetin binding site and tryptophan 214 in HSA (r) was estimated to be $2.6 \times 10^{8} \mathrm{~s}^{-1}$ and $22.8 \AA$ respectively. The corresponding values for BSA were estimated to be $2.1 \times 10^{8} \mathrm{~s}^{-1}$ and $23.7 \AA$ respectively

The fluorescence lifetime measurements for these samples were also made. However, as discussed above, multiple exponential decays were observed. In presence of quercetin, all the lifetime components showed decrease and their relative contributions also varied. As a result, although the average lifetimes decreased, the energy transfer efficiency under these conditions was much less than that determined by steady state measurements. Since it is not clear which of the lifetime components is actually responsible for energy transfer, lifetime data are not used to calculate distance measurements.

\section{Conclusions}

Using absorption and fluorescence spectroscopy, the association constant and number of binding sites for quercetin to serum albumins and HSA and BSA were determined. Binding constants of $\sim 10^{4} \mathrm{M}^{-1}$ and single binding sites were estimated for these systems. The large binding constant values suggest that quercetin binds to the high affinity binding sites of albumins. Good agreement in these estimations by absorption and fluorescence is observed for HSA, while the values differ by an order of magnitude for BSA due to the presence of two tryptophans. Timeresolved fluorescence measurements show multi exponential decay for the protein fluorescence and the average lifetimes decrease in the presence of quercetin. Applying Förster's theory of energy transfer, the distance between quercetin binding site and the tryptophan 214 in HSA and tryptophan 212 in BSA were calculated to be 22.8 and $23.7 \AA$ respectively. These values are slightly lower than those reported by $\mathrm{Bi}$ et $a l .{ }^{31}$ However, our estimated parameters appear to be more accurate than those of $\mathrm{Bi}$ et al, as the present studies employed samples prepared by extensive dialysis, where unbound quercetin is absent, therefore errors in the estimation $J$ value are minimum. From these results it is proposed that the most probable binding site of quercetin in HSA is where the single tryptophan is present, i.e. in domain IIA, which is close to that proposed for other known biomolecules like prodan and warfarin. ${ }^{4,17}$ This loca- 
tion is in agreement with the proposed site of binding for quercetin and flavonoids in general by several other methods. Thus our fluorescence energy transfer studies have provided direct evidence for the binding characteristics of quercetin to serum albumins.

\section{Acknowledgements}

The authors are grateful to Dr T Mukherjee for encouragement and support and Dr H S Mishra, Molecular Biology Division for help with the dialysis experiments.

\section{References}

1. Peters T 1985 Serum albumin. Advances in protein chemistry (New York: Academic Press) 37161

2. Carter D C and Ho J X 1994 Serum albumin. Structure. Advances in protein chemistry (New York: Academic Press) 45153

3. Steinhard J, Krijn J and Leidy J G 1971 Biochemistry 104005

4. Kragh-Hansen U 1981 Pharmacol. Rev. 3317

5. Kumar C V and Buranaprapuk A 1999 J. Am. Chem. Soc. 1214262

6. Jaiswal R, Khan M A and Mussarat J 2002 J. Photochem. Photobiol. B67 163

7. Filipe $\mathrm{P}$, Morlière $\mathrm{P}$, Patterson L K, Hug G L, Mazière C, Freitas J P, Fernandes A and Santus R 2002 Biochim. Biophys. Acta 1572150

8. Jacobsen J and Brodersen R 1983 J. Biol. Chem. 258 6319

9. Reed R G 1977 J. Biol. Chem. 2527483

10. Moreno F, Cortijo M and Gonzalez-Jimenez J 1999 Photochem. Photobiol. 698

11. Feng $X \mathrm{Z}$, Lin Z, Yang L J, Wang C and Bai C L 1998 Talanta 471223

12. Gelamo E L and Tabak M 2000 Spectrochim. Acta A56 2255
13. Pal B, Bajpai P K and Basu Baul T S 2000 Spectrochim. Acta A56 2453

14. Panjehshahin M R, Bowmer C J and Yates M S 1989 Biochem. Pharmacol. 38155

15. Vorum H, Fisker K and Brodersen R 1994 Biochim. Biophys. Acta 1205178

16. Kragh-Hansen U 1985 Biochem. J. 225629

17. Krisnakumar S S and Panda D 2002 Biochemistry 41 7443

18. Barik A, Priyadarsini K I and Mohan H 2003 Photochem. Photobiol. 77597

19. Amisha Kamal J K and Behere D V 2002 J. Biol. Inorg. Chem. 7273

20. He X M and Carter D C 1992 Nature (London) 358209

21. Mikusinska-Planner A and Surma M 2000 Spectrochim. Acta A56 1835

22. Walle T 2004 Free Radical Biol. Med. 36829

23. Williams R J, Spencer J and Rice-Evans C 2004 Free Radical Biol. Med. 36838

24. Boulton D W, Walle U K and Walle T 1998 J. Pharm. Pharmacol. 5043

25. Dangles O, Dufour C, Manach C, Morand C and Remesy C 2001 Method Enzymol. 335319

26. Manach C, Morand C, Texier O, Favier M L, Agullo G, Demigne C, Regerat F and Remesy C 1995 J. Nutr. 1251911

27. Zsila F, Bikádi Z and Simonyi M 2003 Biochem. Pharmacol. 65447

28. Guharay J, Sengupta B and Sengupta P 2001 Proteins 4375

29. Sengupta B and Sengupta P K 2002 Biophys. Biochem. Res. Commun. 299400

30. Sengupta B and Sengupta P K 2003 Biopolymers 72 427

31. Bi S, Din, L, Tian Y, Son D, Zhou X, Liu X and Zhang H 2004 J. Mol. Struct. 70337

32. Dufour C and Dangles O 2005 Biochim. Biophys. Acta 1721164

33. Sytnik A and Litvinyuk I 1996 Proc. Natl. Acad. Sci. USA 9312959

34. Lakowicz J R 1999 Principles of fluorescence spectroscopy (New York: Kluwer Academic/Plenum)

35. O'Conor D V and Phillips D 1984 Time correlated single photon counting (New York: Academic Press) 